UNIVERSIDADE DE BRASÍLIA

FACULDADE DE ECONOMIA, ADMINISTRAÇÃO E CONTABILIDADE

PROGRAMA DE PÓS-GRADUAÇÃO EM ECONOMIA

PREORDENS REGULARES E INDIFERENÇA COMPORTAMENTAL

MAURICIO ALMEIDA COURI RIBEIRO

DISSERTAÇÃO DE MESTRADO EM ECONOMIA

BRASÍLIA/DF

JANEIRO/2016 


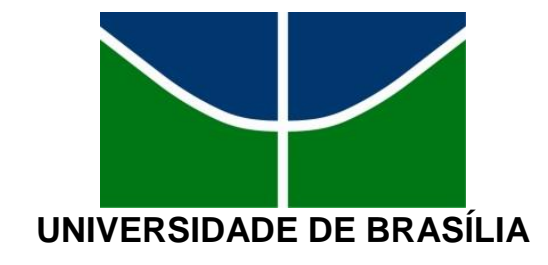

FACULDADE DE ECONOMIA, ADMINISTRAÇÃO E CONTABILIDADE PROGRAMA DE PÓS-GRADUAÇÃO EM ECONOMIA

PREORDENS REGULARES E INDIFERENÇA COMPORTAMENTAL

MAURICIO ALMEIDA COURI RIBEIRO

ORIENTADOR: GIL RIELLA

DISSERTAÇÃO DE MESTRADO EM ECONOMIA

BRASÍLIA/DF

JANEIRO/2016 
Aos meus avós maternos, Joca e Clea, Pela minha capacidade de existir E de saber amar 


\section{Agradecimentos}

Ao meu orientador, Gil Riella.

Aos meus professores e amigos Daniel Cajueiro, Ricardo Araújo e José Guilherme Lara Resende.

Ao professor Leandro Nascimento pelas matérias que ele ministrou no mestrado.

Aos meus pais e ao meu irmão.

À Maria Aparecida de Ramalho Bastos.

A todos os que acreditaram e, principalmente, a todos os que duvidaram de $\operatorname{mim}$.

Aos meus amigos, e ponto.

A meus inimigos. e pronto. 
"The good life is one inspired by love And guided by knowledge." Bertrand Russell 


\title{
PREORDENS REGULARES E INDIFERENÇA COMPORTAMENTAL
}

\begin{abstract}
RESUMO
Nessa dissertação, são discutidos alguns aspectos dos fundamentos teóricos da escolha com preferências incompletas formulados por Eliaz e Ok (2006). Nosso objetivo é clarificar a condição de regularidade para preordens introduzida por esses autores, mostrando que ela não impõe nenhuma restrição se apenas estivermos interessados em racionalizar uma correspondência de escolha. Porém, se estivermos interessado em capturar outros aspectos observáveis do comportamento de escolha de um agente, por exemplo, a noção de cincomparabilidade de Eliaz e Ok (2006) ou a noção de indiferença comportamental introduzida nesta dissertação, então a única opção é usar uma preordem regular. Argumentamos também que o Axioma Fraco da Não-Inferioridade Revelada (AFNIR) introduzido por Eliaz e Ok (2006) é muito forte se quisermos caracterizar a racionabilidade de uma correspondência de escolha por uma preordem possivelmente incompleta. Finalmente, como já foi mencionado acima, nós introduzimos a noção de indiferença comportamental e argumentamos que ela é mais geral do que a noção de Eliaz e Ok (2006) de c-incomparabilidade, sendo, ao mesmo tempo, complementar a esta dentro do framework dos autores.
\end{abstract}

Palavras-chave: Preferências Incompletas, Escolha Racional, Não-inferioridade, Preordens Regulares, Indiferença Comportamental. 


\title{
PREORDENS REGULARES E INDIFERENÇA COMPORTAMENTAL
}

\begin{abstract}
In this thesis, we discuss some aspects of Eliaz and Ok (2006)'s choice theoretical foundations of incomplete preferences. Our aim is to clarify their regularity condition for preorders, showing that, as far as rationalization of a choice correspondence alone is concerned, no further restriction is imposed by requiring the preorder to be regular. However, if one is also interested in capturing other observable aspects of the individual's choice procedure, such as Eliaz and Ok's notion of c-incomparability or the notion of behavioral indifference introduced in this paper, then the only option is to use a regular preorder. We also argue that their Weak Axiom of Revealed Non-inferiority (WARNI) is too strong a property if our aim is to characterize the rationalizability of a choice correspondence by a (possibly incomplete) preorder. Finally, as we have mentioned above, we introduce the notion of behavioral indifference and argue, not only that Eliaz and Ok's notion of c-incomparability (observable incomparability) can be derived from it, but also that it has a wider range of applicability than their notion of observable incomparability.
\end{abstract}

Keywords: Incomplete Preferences, Rational Choice, Non-inferiority, Regular Preorders, Behavioral Indifference. 


\section{Sumário}

1 Introdução 2

2 Definições Preliminares $\quad 4$

2.1 Correspondências de Escolha . . . . . . . . . . . . . . . . . 4

2.2 Relações Binárias . . . . . . . . . . . . . . . . . . 4

2.3 Racionalização de Correspondências de Escolha e Indiferença Comporta-

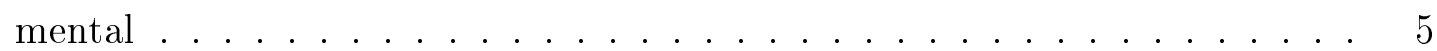

3 Preordens Regulares e Racionalização 9

4 Axiomas de Racionalidade e Teoremas de Representação 12

5 Conclusão $\quad 15$ 


\section{Capítulo 1}

\section{Introdução}

Preferências Incompletas é uma das principais áreas da Teoria da Decisão contemporânea. Um dos artigos mais importantes da área é intitulado "Indifference or indecisiveness? Choice theoretic foundations of incomplete preferences", escrito por Kfir Eliaz e Efe Ok em 2006. Esse artigo tem dois objetivos principais. Em primeiro lugar, os autores buscam expandir o escopo de aplicação do paradigma maximizador, flexibilizando o Axioma Fraco da Preferência Revelada (AFNIR). Por outro lado, eles propõe um framework que permite diferenciar entre indiferença e incompletude baseado no comportamento observável dos agentes.

Neste trabalho, tentaremos clarificar, e até certo ponto melhorar, alguns aspectos da análise de Eliaz e Ok (2006).

No Capítulo 2, introduziremos as definições necessárias.

No Capítulo 3, argumentaremos que, ao invés de tomar a noção proposta por esses autores de incomparabilidade observável (ou cincomparabilidade) como primitiva, deveríamos começar com a noção de indiferença comportamental, que será definida. Apesar de que, no setup de Eliaz e Ok (2006), essas noções são complementares em um sentido bem definido, acreditamos que a noção de indiferença comportamental é mais fundamental e tem um maior escopo de aplicação. Substanciaremos essa afirmação mostrando alguma das implicações que essa noção tem para certos modelos de decisão, a saber os models of behavior.

No Capítulo 4, analisaremos a propriedade de regularidade para preordens, introduzida por Eliaz e Ok (2006). Essa propriedade é essencial se quisermos que a noção de indiferença comportamental se traduza como indiferença pela preordem utilizada para racionalizar uma dada correspondência de escolha. Mostraremos, em particular, que, se estivermos apenas interessados na racionalização de uma correspondência de escolha, a regularidade não impõe nenhuma restrição adicional: ela é equivalente ao um certo tipo de maximalidade da preordem usada no processo de representação da correspondência de escolha. No entanto, se quisermos não só racionalizar, mas também em capturar certos aspectos do comportamento de escolha de um agente, e. $g$. a noção de indiferença 
comportamental, então exigir que a preordem seja regular é essencial.

Por fim, no Capítulo 5, demonstraremos que, se o nosso objetivo é obter uma caracterização de racionabilidade de uma correspondência de escolha por uma preordem possivelmente incompleta, então o Axioma Fraco da Preferência Revelada de Eliaz e Ok (2006), é uma propriedade muito forte e pode ser enfraquecida. Diante disso, propomos um relaxamento conveniente do postulado que nos dá uma caracterização completa da classe de correspondências de escolha que podem ser racionalizada por uma preordem possivelmente incompleta. 


\section{Capítulo 2}

\section{Definições Preliminares}

Seguiremos o setup sugerido por Eliaz and Ok (2006).

\subsection{Correspondências de Escolha}

Seja $X$ um conjunto arbitrário. Interpretaremos $X$ como sendo o conjunto de todas as alternativas mutuamente excludentes.

Definição 1. Um campo de escolha sobre $X$, denotado por $\Omega_{X}$, é um subconjunto de $2^{X} \backslash\{\emptyset\}$ com as seguintes propriedades:

(I) $\{x\} \in \Omega_{X}$, para todo $x \in X$;

(II) $\bigcup_{i=1}^{n} A_{i} \in \Omega_{X}$ sempre que $A_{i} \in \Omega_{X}, i=1,2, \ldots, n, n \in \mathbb{N}$.

Interpretaremos $\Omega_{X}$ como o conjunto de todos os problemas de escolha de um determinado agente. Note que, pela definição, todo subconjunto finito de $X$ está em $\Omega_{X}$. Denotaremos o par $\left(X, \Omega_{X}\right)$ um espaço de escolha sempre que $|X| \geqslant 3$.

Definição 2. Dado um espaço de escolha $\left(X, \Omega_{X}\right)$, definiremos uma correspondência de escolha sobre $\Omega_{X}$ como uma correspondência $c: \Omega_{X} \rightrightarrows X$ que satisfaz:

(I) $c(S) \neq \emptyset$, para todo $S \in \Omega_{X}$;

(II) $c(S) \subseteq S$, para todo $S \in \Omega_{X}$.

\subsection{Relações Binárias}

Dado qualquer conjunto $X$, uma relação binária sobre $X$ é simplesmente um conjunto não-vazio de $X \times X$. Adotaremos a notação padrão $x \succsim y$ para representar o fato de que $(x, y) \in \succsim$. Definiremos a parte simétrica de uma relação $\succsim$ por $\backsim:=\{(x, y) \in$ $X \times X: x \succsim y$ and $y \succsim x\}$. A parte assimétrica de uma relação $\succsim$ é definida como sendo 
$\succ:=\succsim \backslash \backsim$. Finalmente, diremos que $x$ e $y$ são incomparáveis e escreveremos $x \bowtie y$, quando nem $x \succsim y$ e $y \succsim x$ forem proposições falsas.

Diremos que uma relação binária $\succsim$ é reflexiva quando $x \succsim x$, para todo $x \in X$. Diremos que é transitiva se $x \succsim y$ e $y \succsim z$ implicam $x \succsim z$, para todo $x, y, z \in X$. Diremos que $\succsim$ é quase-transitiva quando sua parte assimétrica, $\succ$, for transitiva, e diremos que $\succsim$ é antissimétrica se $x \succsim y$ e $y \succsim x$ implicarem que $x \backsim y$. Por fim, diremos que $\succsim e ́$ completa se $(x \succsim y$ ou $y \succsim x)$ for verdade, para todo $x, y \in X$.

Ademais, diremos que $\succsim$ é uma a preordem, ou uma relação de preferência sempre que ela for reflexiva e transitiva. Uma ordem parcial é simplesmente uma preordem antissimétrica.

Para qualquer preordem $\succsim \subseteq X \times X$ e $\emptyset \neq S \subseteq X$, o conjunto de elementos $\succsim$ maximais de $S$ será denotado por $\operatorname{MAX}(S, \succsim)$, isto é

$$
\operatorname{MAX}(S, \succsim):=\{x \in S: \text { não existe } y \in S \text { tal que } y \succ x\}
$$

Também trabalharemos com uma definição um pouco distintas de maximalidade, denotada por $\max (S, \succsim)$, e que vem dada pela expressão

$$
\max (S, \succsim):=\{x \in S: x \succsim y, \text { for all } y \in S\}
$$

Finalmente, diremos que uma relação binária $\succsim$ é uma extensão de uma relação binária $\succsim$ se $\hat{\succsim} \supseteq \succsim$ e $\hat{\succ} \supseteq \succ$.

\subsection{Racionalização de Correspondências de Escolha e Indiferença Comportamental}

Seja $\left(X, \Omega_{X}\right)$ um espaço de escolha e $c$ um correspondência de escolha sobre $\Omega_{X}$. Sob que circunstâncias diremos que as escolhas de um agente, expressas por $c$, podem ser interpretadas como se emergisse do processo de maximização de suas preferências subjacentes? A resposta dessa pergunta depende, é claro, da noção de maximização que é utilizada. Por um tempo, a Teoria da Escolha assumiu que as preferências subjacentes de um agente deveriam ser representadas por uma preordem completa. Nesse espírito, temos a seguinte definição:

Definição 3. Dada uma correspondência de escolha $c$ sobre $\Omega_{X}$, diremos que c é racionalizada por uma preordem completa $\succsim \subseteq X \times X$ se, para todo $S \in \Omega_{X}$,

$$
c(S)=\max (S, \succsim)
$$

Logo, a racionalização por uma preordem completa requer que, em cada conjunto de escolha $S \in \Omega_{X}$, se $x \in S$ é escolhido em $S$, então devemos ter que $x$ é fracamente prefe- 
rível a todo $y \in S$. Apesar de sensatez dessa definição, não é difícil ver que ela é bastante restritiva. Isso se deve à exigência de que a preordem utilizada no processo de racionalização seja completa, o que exclui muitas preordens possíveis como possíveis candidatas para representar as preferências subjacentes dos indivíduos. Eliaz and Ok (2006) perceberam o quão restritiva era essa definição e, por isso, argumentaram que o paradigma maximizador poderia ser expandido enfraquecendo o conceito de racionalização. Dessa perspectiva, considere a seguinte definição:

Definição 4. Seja $\left(X, \Omega_{X}\right)$ um espaço de escolha, e $c$ uma correspondência de escolha em $\Omega_{X}$. Diremos que $c$ é racionalizada por uma preordem $\succsim \subseteq X \times X$ se, para todo $S \in \Omega_{X}$,

$$
c(S)=\operatorname{MAX}(S, \succsim)
$$

É fácil ver que a racionalização por uma preordem é um conceito mais geral do que racionalização por uma preordem completa. De fato, para $x \in S$ ser escolhido em $S$, apenas exigimos que $x$ não seja estritamente dominado por algum $y \in S$. Nesse sentido, o espectro de comportamentos de escolha que podemos chamar de racional é consideravelmente expandido.

Devemos notar ainda que para o conceito de racionalização descrito na Definição 4, a única coisa que importa é a parte estrita da relação $\succsim$. Isso implica que, só estivermos preocupados com a racionalização, muitos tipos de relações binárias são candidatas no processo de racionalização de preferências. Podemos, por exemplo, usar uma relação binária quase-transitiva.

Contudo, ao considerarmos o processo de racionalização, poderíamos estar interessados que a relação de preferência utilizada espelhasse outros aspectos do comportamento de escolha observável do agente. Ou seja, seria desável que a preordem utilizada para racionalizar a correspondência de escolha do agente preservasse outros atributos comportamentais. Mas quais seriam esses outros atributos observáveis? Nessa seção, focaremos em dois desses atributos: a noção de Eliaz e Ok (2006) de $c$-incomparabilidade e a noção de indiferença comportamental, que será definida abaixo.

Até Eliaz and Ok (2006), uma questão fundamental na Teoria da Escolha era como distinguir, de forma significativa, entre indiferença e incomparabilidade baseado no comportamento de escolha observável do agente. É claro que essa questão só pode ser respondida se, primeiro, decidirmos quando diremos que o agente julga que duas alternativas são incomparáveis (ou indiferentes). Mas isso não é tudo: a definição deve ser dada considerando somente o seu comportamento de escolha observável, $i$. e. em termos da correspondência de escolha $c$. Em resumo: necessitamos de uma definição observável de incomparabilidade (ou de indiferença).

Eliaz and Ok (2006) propuseram uma definição razoável de indiferença comportamental. Eles a denominaram de c-incomparabilidade (ver Eliaz and Ok, 2006). No entanto, 
argumentamos a noção de indiferença comportamental é mais fundamental do que noção de incomparabilidade observável, no sentido que é ela é aplicável a uma maior classe de modelos de decisão. Com isso em mente, propomos a seguinte definição:

Definição 5. Seja $\left(X, \Omega_{X}\right)$ um espaço de escolha, e $c$ uma correspondência de escolha sobre $\Omega_{X}$. Diremos que $x, y \in X$ são comportamentalmente indiferentes se, para todo $S \in \Omega_{X}$,

(I) $x \in c(S \cup\{x\})$ se, e somente se, $y \in c(S \cup\{y\})$;

(II) $c(S \cup\{x\}) \backslash\{x, y\}=c(S \cup\{y\}) \backslash\{x, y\}$.

Intuitivamente, essa definição tem uma interpretação simples: o agente considera $x, y \in X$ comportamentalmente diferentes quando ele os considera indistinguíveis do ponto de vista do seu comportamento de escolha. É como se o agente considerasse $x$ e $y$ idênticos quando estivesse decidindo.

Observação 1. É fácil mostrar que, em Eliaz and Ok (2006), dado que $c(\{x, y\})=\{x, y\}$, ou $x$ e $y$ são $c$-incomparáveis ou eles são comportamentalmente indiferentes. Poderíamos, então, dizer que, em Eliaz and Ok (2006), se restringirmos nossa atenção ao conjunto $\{(x, y) \in X \times X: c(\{x, y\})=\{x, y\}\}, c$-incomparabilitdade é não-indiferença comportamental, e indiferença comportamental é $c$-não-incomparabilidade. Então, no framework de Eliaz e Ok, podemos postular a noção de indiferença comportamental e derivar dela a noção de $c$-incomparabilidade.

Como já sugerimos ao introduzir a noção de indiferença comportamental, acreditamos que essa noção é mais fundamental do que a noção de incomparabilidade observável. Em primeiro lugar, como acabamos de argumentar, no framework de Eliaz e Ok, esta pode ser derivada daquela. Mais ainda, o conceito de indiferença comportamental tem um espectro de aplicação maior. Ilustremos esse pontos analisando quais as implicações do conceito de indiferença comportamental para um conjunto de modelos de decisão conhecidos como models of behavior.

Seguindo Cherepanov et al. (2013), diremos que um par $(\succsim, \psi)$ é um model of behavior sobre um espaço de escolha $\left(X, \Omega_{X}\right)$ se $\succsim$ é uma preordem completa sobre $X$ e $\psi: \Omega_{X} \rightrightarrows X$ satisfaz $\emptyset \neq \psi(S) \subseteq S$, para todo $S \in \Omega_{X}$. Ademais, diremos que uma correspondência de escolha $c$ em $\Omega_{X}$ é racionalizada por um model of behavior $(\succsim, \psi)$ se $c(S)=\max (\psi(S)$, $)$, para todo $S \in \Omega_{X}$. Temos, então, o seguinte teorema.

Teorema 1. Seja $\left(X, \Omega_{X}\right)$ um espaço de escolha, e c uma correspondência de escolha sobre $\Omega_{X}$ que é racionalizada por um model of behavior $(\succsim, \psi)$. Mais ainda, suponha que $x, y \in X$ são tais que $x \backsim y$ e que, para todo $S \in \Omega_{X}$,

(1) $x \in \psi(S \cup\{x\})$ se, e somente se, $y \in \psi(S \cup\{y\})$; 
(2) $\psi(S \cup\{x\}) \backslash\{x, y\}=\psi(S \cup\{y\}) \backslash\{x, y\}$.

Então, $x$ e y são comportamentalmente indiferentes. De forma conversa, se c é racionalizável por um model of behavior $(\succsim, \psi)$, então existe $\phi: \Omega_{X} \rightrightarrows X$ tal que $(\succsim, \phi)$ é um outro model of behavior que racionaliza $c$ e, para todo par $x, y \in X$ comportamentalmente indiferentes, temos que $x \backsim y$, e (1) e (2) acima são verdadeiras com $\psi$ substituído por $\phi$.

Demonstração. Seja $(\succsim, \psi)$ um model of behavior e $c$ uma correspondência de escolha em $\Omega_{X} \operatorname{com} c(S)=\max (\psi(S), \succsim)$, para todo $S \in \Omega_{X}$. Fixe $x, y \in X \operatorname{com} x \backsim y \mathrm{e}$ satisfazendo as condições (1) e (2) para todo $S \in \Omega_{X}$. Devemos provae que $x$ e $y$ são comportamentalmente indiferentes. Para isso, fixe $S \in \Omega_{X}$. Usando (1), (2) e o fato que $x \backsim y$, chegamos na seguinte sequência de equivalências:

$$
\begin{aligned}
x \in c(S \cup\{x\}) & \Leftrightarrow x \in \psi(S \cup\{x\}) \text { e } x \succsim z, \text { para todo } z \in \psi(S \cup\{x\}) \\
& \Leftrightarrow y \in \psi(S \cup\{y\}) \text { e } y \succsim z \text { para todo } z \in \psi(S \cup\{x\}) \\
& \Leftrightarrow y \in \psi(S \cup\{y\}) \text { e } y \succsim z, \text { para todo } z \in \psi(S \cup\{y\}) \\
& \Leftrightarrow \quad y \in c(S \cup\{y\}) .
\end{aligned}
$$

Logo, $x \in c(S \cup\{x\})$ se, e somente se, $y \in c(S \cup\{y\})$. Por um argumento similar, estabelecemos que $c(S \cup\{x\}) \backslash\{x, y\}=c(S \cup\{y\}) \backslash\{x, y\}$. Portanto, concluímos que $x$ e $y$ são comportamentalmente indiferentes. Para estabelecer a conversa, suponha que $c$ é racionalizada por um model of behavior $(\psi, \succsim)$. Defina $\phi(S):=c(S)$, para todo $S \in \Omega_{X}$. É facilmente visto que $(\succsim, \phi)$ ainda racionaliza $c$. Suponha, então, que $x$ e $y$ são comportamentalmente indiferentes. Que, para todo $S \in \Omega_{X}$, (1) e (2) com $\psi$ substituído por $\phi$ deve valer é uma consequência direta da Definição 5. Mais ainda, segue-se do fato de que $x$ e $y$ são comportamentalmente indiferentes que $\max (\phi(\{x, y\}), \succsim)=c(\{x, y\})=$ $\{x, y\}$. Logo, $x \backsim y$.

Como o Teorema 1 mostra, a noção de indiferença comportamental tem implicações interessantes para models of behavior. Em particular, ela implica que, se a correspondência de escolha $c$ pode ser racionalizada por um model of behavior, então sempre podemos construir um outro model of behavior que ainda representa $c$ e tal que a noção de indiferença comportamental é intuitivamente capturada pelos elementos do modelo. 


\section{Capítulo 3}

\section{Preordens Regulares e Racionalização}

Como discutimos anteriormente, quando racionalizamos uma correspondência de escolha $c$ por uma preordem possivelmente incompleta $\succsim$, podemos estar interessados que $\succsim$ também capture outros traços observáveis do comportamento de escolha individual. Em particular, podemos demandar que $\succsim$ capture as noções de indiferença e incomparabilidade comportamentais. Acontece que se representarmos a correspondência de escolha $c$ com uma preordem $\succsim$ arbitrária, esse não vai ser o caso em geral. Para lidar com esse problema, Eliaz and Ok (2006) introduziram a seguinte definição:

Definição 6. A preordem $\succsim$ em um conjunto não vazio $X$ é dita regular se, para qualquer $x, y \in X \operatorname{com} x \bowtie y$, existe um $z \in X$ tal que ou $x \bowtie z$ e $z, y$ são estritamente ordenados por $\succsim$, ou $y \bowtie z$ e $z, x$ são estritamente comparáveis por $\succsim$.

Intuitivamente, a regularidade impõe que, para que $x$ e $y$ sejam considerados incomparáveis, deve existir uma "prova" desse fato, no sentido de que deve existir um $z$ que é estritamente comparável a algum desses elemetnos, mas não ao outro. Acontece que regularidade é exatamente a propriedade que garante que a preordem $\succsim$ captura precisamente nossa noção de indiferença comportamental. Formalmente, temos o seguinte resultado:

Lema 1. Seja $\left(X, \Omega_{X}\right)$ um espaço de escolha, e c uma correspondência de escolha sobre $\Omega_{X}$ que é racionalizada por uma preordem $\succsim$. Para todo par de alternativas $x$ e y em $X$, se $x \backsim y$, então $x$ e y são comportamentalmente indiferentes. Se, além disso, $\succsim e ́$ regular, então a conversa também é verdade. Ou seja, para todo $x, y \in X, x$ e y são comportamentalmente indiferentes se, e somente se, $x \backsim y$.

Demonstração. É fácil checar que se $c$ é uma correspondência de escolha racionalizada por uma preordem $\succsim$, então $x \backsim y$ implica que $x$ e $y$ são comportamentalmente indiferentes. Iremos apenas mostrar que se $\succsim$ é regular, então a afirmação conversa também é verdade. Para ver isso, suponha que $\succsim$ seja uma preordem regular que racionaliza uma correspondência de escolha $c$ e escolha um par $x, y \in X$ comportamentalmente indiferentes. Logo, devemos ter que ou $x \backsim y$ ou $x \bowtie y$. Fixe $z \in X \operatorname{com} z \bowtie x$. Pela representação de $c$, isso 
implica que $c(\{x, z\})=\{x, z\}$. Como $x$ e $y$ são comportamentalmente indiferentes, isso agora implica que $c(\{y, z\})=\{y, z\}$. Pela representação de $c$, devemos ter que $y \backsim z$ or $y \bowtie z$. Um raciocínio simétria mostra que, sempre que $z \in X$ é tal que $y \bowtie z$, então não podemos ter que $x$ e $z$ são estritamente ordenados por $\succsim$. A regularidade, agora, implica que $x \bowtie y$. Portanto, $x \backsim y$.

Acontece que requerer regularidade não impõe restrição adicional na possibilidade de racionalização de uma correspondência de escolha por uma preordem, $i$. e. sempre que uma correspondência de escolha $c$ possa ser racionalizada por uma preordem $\succsim$, então existe uma única preordem regular $\hat{\succsim}$ que também racionaliza $c$. Esse fato é uma consequência simples do seguinte resultado:

Lema 2. Seja $X$ um conjunto qualquer. Então, para toda preordem $\succsim \subseteq X \times X$, existe

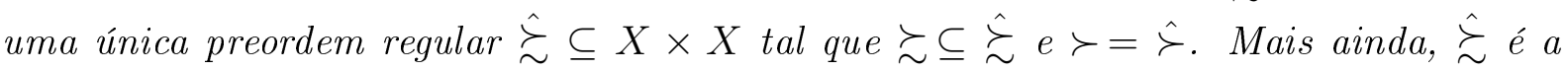
extensão transitiva maximal de $\succsim$ tal que $\succ=\hat{\succ}$.

Demonstração. Fixe qualquer preordem $\succsim \subseteq X \times X$. Defina a relação binária $\hat{\succsim} \subseteq X \times X$ por $x \hat{\succsim} y$ se, e somente se, $x \succsim y$ ou $x \bowtie y$ e, para todo $z \in X, z \succ x \Leftrightarrow z \succ y$

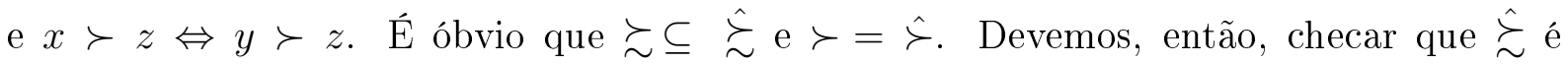
uma preordem regular. É óbvio também que $\hat{\succsim}$ is reflexiva. Ademais, por construção, $\hat{\succsim}$ é regular, pois $\hat{\succsim}$ pode ser vista como uma extensão de $\succsim$ na qual os pares que são considerados incomparáveis por $\succsim$ e que não satisfaz a condição imposta pela regularidade são, agora, considerados indiferentes por $\hat{\succsim}$. Devemos, então, mostrar que $\hat{\succsim}$ é transitiva. Fixe $x, y$ e $z$ em $X$ com $x \hat{\succsim} y \hat{\succsim} z$. Se $x \hat{\succ} y$ ou $y \hat{\succ} z$, então obtemos que $x \hat{\succ} z$ como uma consequência imediata da transitividade de $\succsim$ e da definição de $\hat{\succsim}$. Suponha, então, que $x \hat{\wedge} y \hat{\wedge} z$. Pela nossa última observação, não podemos ter que $z \succ x$ (e, consequentemente, $z \hat{\succ} x$ ), pois isso implicaria que $z \hat{\succ} y$. Logo, devemos apenas considerar o caso que $x \bowtie z$. Fixe qualquer $w \in X$. Pela transitividade de $\succsim$ e a definição de $\hat{\succsim}$, devemos ter que $w \succ x \Leftrightarrow w \succ y \Leftrightarrow w \succ z$ e $x \succ w \Leftrightarrow y \succ w \Leftrightarrow z \succ w$. Como $w$ foi escolhido arbitrariamente, concluímos que $x \hat{\sim} z$. Logo, $\hat{\succsim}$ é transitiva.

Para estabelecer a unicidade de $\hat{\succsim}$, considere outra preordem $\succsim$ tal que $\succsim \subseteq \succsim \mathrm{e}$ $\succ=\succ$. Fixe um par $x, y \in X \operatorname{com} x \backsim y$. Como $\succ=\succ=\hat{\succ}$, devemos ter que $x \hat{\sim} y$ ou $x \hat{\bowtie} y$. Mais ainda, é claro que $z \succ x \Leftrightarrow z \succ y$ e $x \succ z \Leftrightarrow y \succ z$, para todo $z \in X$. Como $\hat{\succsim}$ é regular e $\hat{\succ}=\succ$, devemos ter $x \hat{n} y$. Isso mostra que $\backsim \subseteq \hat{\sim}$. Como $\succsim$ também é regular, podemos inverter os papéis de $\succsim$ e $\hat{\succsim}$ e concluir que $\hat{\sim} \subseteq \tilde{n}$. Isso mostra que $\hat{\succsim}$ é a única preordem que $\succsim \subseteq \hat{\succsim} \mathrm{e} \succ=\hat{\succ}$. Incidentalmente, essa última observação mostra que $\hat{\succsim}$ é a extensão transitiva máxima tal que $\succsim \subseteq \hat{\succsim} \mathrm{e} \succ=\hat{\succ}$.

Uma implicação imediata do Lema 2 é que, sempre que a correspondência de escolha $c$ sobre $\Omega_{X}$ possa ser racionalizada por uma preordem, então existe uma única preordem regular que também racionaliza $c$. Logo, o Lema 2 corrobora nossa afirmação que a 
regularidade é completamente não restritiva se estivermos apenas interessados com a racionalização. Ainda mais, isso implica que uma correspondência de escolha racionalizável pode sempre ser racionalizada por uma preordem que identifica a indiferença comportamental com a sua parte simétrica. Finalmente, o Lema 2 também nos ajuda a esclarecer o conceito de regularidade. Note que ela implica que, entre toda as preordens que compartilham da mesma parte estrita, existe apenas uma que é regular e ela é justamente a mais completa entre elas. Intuitivamente, demandar regularidade é o mesmo que demandar que, sempre que possamos chamar duas alternativas de indiferentes sem violar a transitividade, assim o façamos.

Exemplo 1. Seja $X:=\{x, y, z\}, \Omega_{X}:=2^{X} \backslash\{\emptyset\}$ e considere a correspondência de escolha $c$ sobre $\Omega_{X}$ dada por $c(\{x, y, z\})=\{x, y\}, c(\{x, y\})=\{x, y\}, c(\{x, z\})=\{x\}$ e $c(\{y, z\})=$ $\{y\}$. Note que $x$ e $y$ são comportamentalmente indiferentes. Agora, defina duas preordens $\succsim_{1}$ e $\succsim_{2}$ sobre $X$ por

1) $x \sim_{1} y \succ_{1} z$;

2) $x \bowtie_{2} y, x \succ_{2} z, y \succ_{2} z$.

É fácil ver que $c(S)=\operatorname{MAX}\left(S, \succsim_{1}\right)=\operatorname{MAX}\left(S, \succsim_{2}\right)$, para todo $S \in \Omega_{X}$. Entretanto, $\succsim_{1}$ é regular, enquanto $\succsim_{2}$ não o é. De acordo com $\succsim_{1}, x \sim_{1} y$, i.e., dois elementos comportamentalmente indiferentes são indiferentes por $\succsim_{1}$, enquanto o mesmo não ocorre com $\succsim_{2}$.

Exemplo 2. Seja $X:=\{x, y, w, z\}, \Omega_{X}:=2^{X} \backslash\{\emptyset\}$ e considere a correspondência de escolha $c$ em $\Omega_{X}$ dada por $c(S)=\{x\}$ para todo $S \in \Omega_{X} \operatorname{com} x \in S, c(\{y, w, z\})=$ $c(\{y, w\})=\{y, w\}, c(\{y, z\})=\{z\}$ e $c(\{w, z\})=\{w, z\}$. Defina, agora, a preordem regular $\succsim_{1}$ e relação binária completa e quase-transitiva $\succsim_{2}$ sobre $X$ por

1) $x \succ_{1} y \succ_{1} z, x \succ_{1} w, y \bowtie_{1} w, \mathrm{e} z \bowtie_{1} w$;

2) $x \succ_{2} y \succ_{2} z, x \succ_{2} w, y \sim_{2} w$ e $z \sim_{2} w$

É fácil checar que $c(S)=\operatorname{MAX}\left(S, \succsim_{1}\right)=\operatorname{MAX}\left(S, \succsim_{2}\right)$, para todo $S \in \Omega_{X}$. Entretanto, enquanto $z, w$ e $y, w$ são comportamentalmente indiferentes, $y \sim_{2} w$ e $z \sim_{2} w$. 


\section{Capítulo 4}

\section{Axiomas de Racionalidade e Teoremas de Representação}

A propriedade mais fundamental da Teoria da Escolha é o Axioma Fraco da Preferência Revelada (AFPR).

Axioma 1. (AFPR) Para todo $S \in \Omega_{X}$ e $y \in S$, se existe um $x \in c(S)$ tal que $y \in c(T)$ para algum $T \in \Omega_{X}$ com $x \in T$, então $y \in c(S)$.

É bem conhecido o fato de que uma correspondência de escolha $c$ pode ser racionalizada por uma preordem completa se, e somente se, ela satisfaz AFPR. Formalmente:

Teorema 2. (Teorema Fundamental da Preferência Revelada). Seja $\left(X, \Omega_{X}\right)$ um espaço de escolha e c $: \Omega_{X} \rightrightarrows X$ uma correspondência de escolha. c satisfaz AFPR se, e somente se, existe uma (única) preordem completa $\succsim$ sobre $X$ tal que $c(S)=\max (S, \succsim)$, para todo $S \in \Omega_{X}$.

Como Eliaz and Ok (2006) observam, o apelo descritivo e normativo do AFPR depende da interpretação que damos à correspondência de escolha. Em particular, certos comportamentos violam AFPR, apesar deles poderem ser explicados dentro do paradigma maximizador. Esse é o caso para problemas de escolha que envolvem múltiplos critérios de decisão. Essas observações fizeram com que Eliaz e Ok propusessem uma propriedade mais fraca de racionalidade, que é mais congenial à interpretação que eles dão de $c(S)$ (Eliaz e Ok, 2006). Eles chamam essa propriedade de Axioma Fraco da Não-Inferioridade Revelada (AFNIR).

Axioma 2. (AFNIR) Para todo $S \in \Omega_{X}$ e $y \in S$, se, para cada $x \in c(S)$, existir um $T_{x} \in \Omega_{X}$ com $y \in c\left(T_{x}\right)$ e $x \in T_{x}$, ent $\tilde{a} o \quad y \in c(S)$.

Eliaz and Ok (2006) argumentam que AFNIR é um relaxamento conveniente do AFPR para um modelo que distingue entre superioridade e não-inferioridade revelada. Os autores, então, provam o seguinte resultado: (Eliaz and Ok, 2006, Teorema 2): 
Teorema 3. Seja $\left(X, \Omega_{X}\right)$ um espaço de escolha e c : $\Omega_{X} \rightrightarrows X$ uma correspondência de escolha. Se c satisfaz AFNIR, então existe uma preordem regular não necessariamente completa $\succsim$ sobre $X$ tal que $c(S)=\operatorname{MAX}(S, \succsim)$ para todo $S \in \Omega_{X}$. De forma conversa, se $\Omega_{X}$ incluir todos os conjuntos enumeráveis de $X$ e existir uma preordem $\succsim$ sobre $X$ tal que $c(S)=\operatorname{MAX}(S, \succsim)$, para todo $S \in \Omega_{X}$, então c satisfaz AFNIR.

O resultado de Eliaz e Ok mostra que uma correspondência de escolha que satisfaz AFNIR pode ser racionalizada por uma preordem regular $\succsim$. Entretanto, a conversa só vale quando o espaço de escolha $\Omega_{X}$ incluir todos os subconjuntos enumeráveis de $X$. Isso sugere que, se estivermos interessados na racionalização de uma correspondência de escolha por uma preordem regular não necessariamente completa, AFNIR pode ser uma propriedade muito forte. Em particular, quando consideramos também o fato de que a correspondência de escolha deve ser não vazia para todo problema de escolha, demandar que $\Omega_{X}$ inclua todos os subconjuntos enumeráveis de $X$ parece ser um pouco restritivo. Isso pode ser facilmente remediado por dois enfraquecimentos naturais do AFNIR:

Axioma 3. (FAFNIR) For any $S \in \Omega_{X}$ e $y \in S$, se para todo $x \in S$ existir um $T_{x} \in \Omega_{X}$ tal que $y \in c\left(T_{x}\right)$ e $x \in T_{x}$, então $y \in c(S)$.

Axioma 4. (Transitividade da Escolha Estrita - TES) Para todo $x, y, z \in X$, se $c(\{x, y\})=$ $\{x\}$ e $c(\{y, z\})=\{y\}$, ent $\tilde{a} o c(\{x, z\})=\{x\}$.

Enquanto o AFNIR demanda apenas que $y$ seja revelado não-inferior a todo elemento de $c(S)$ para que $y$ esteja entre os elementos escolhidos de $S$, FFNIR demanda que $y$ seja revelado não-inferior a todo lemento de $S$ para que isso aconteça. É fácil checar que AFNIR implica FAFNIR, mas que a volta não vale. O seguinte exemplo ilustra a diferença entre AFPR, AFNIR e FAFNIR.

Exemplo 3. Seja $X:=\{x, y, z\}$ e considere as seguintes correspondências de escolha sobre $\Omega_{X}$ :

1) $c_{1}(\{x, y, z\})=\{x, y, z\}, c_{1}(\{x, y\})=\{x, y\}, c_{1}(\{x, z\})=\{x, z\}, c_{1}(\{y, z\})=\{y, z\}$;

2) $c_{2}(\{x, y, z\})=\{x, y\}, c_{2}(\{x, y\})=\{x, y\}, c_{2}(\{x, z\})=\{x, z\}, c_{2}(\{y, z\})=\{y\}$;

3) $c_{3}(\{x, y, z\})=\{x\} ; c_{3}(\{x, y\})=\{x\}, c_{3}(\{x, z\})=\{x, z\}, c_{3}(\{y, z\})=\{y\}$.

É fácil checar que $c_{1}$ satisfaz AFPR (e, por isso, satisfaz também AFNIR e FAFNIR). $c_{2}$ não satisfaz AFPR, mas satisfaz AFNIR (e, portanto, satisfaz FAFNIR). E, finalmente, $c_{3}$ satisfaz FAFNIR, mas não satisfaz AFNIR (e, portanto, não pode satisfazer AFPR).

O axioma da Transitividade da Escolha Estrita impõe uma forma natural de transitividade sobre as escolhas nos conjuntos de dois elementos. É fácil chegar que AFNIR implica TES e, portanto, que o par FAFNIR e TES é um enfraquecimento do AFNIR . De fato, como mostra nosso próximo teorema, esse par fornece exatamente o relaxamento que caracteriza a racionalização por uma preordem possivelmente incompleta (e regular). 
Teorema 4. Seja $\left(X, \Omega_{X}\right)$ um espaço de escolha e c : $\Omega_{X} \rightrightarrows X$ uma correspondência de escolha sobre $\Omega_{X}$. c satisfaz FAFNIR e TES se, e somente se, existe uma única preordem regular (não necessariamente completa) $\succsim$ sobre $X$ tal que $c(S)=\operatorname{MAX}(S, \succsim)$, para todo $S \in \Omega_{X}$.

Demonstração. É fácil checar que se existe uma preordem $\succsim$ sobre $X$ tal que $c(S)=$ $\operatorname{MAX}(S, \succsim)$, para todo $S \in \Omega_{X}$, então $c$ satisfaz FAFNIR e TES. Para mostrar a conversa, suponha que $c: \Omega_{X} \rightrightarrows X$ satisfaz FAFNIR e TES. Defina

$$
x \succcurlyeq y \Leftrightarrow c(\{x, y\})=\{x\}
$$

É claro que $\succcurlyeq$ é reflexiva, antissimétrica e, por TES, transitiva. Logo, $\succcurlyeq$ é uma ordem parcial. Fixe $S \in \Omega_{X}$ e $x \in c(S)$. Por FAFNIR, $x \in c(\{x, y\})$ para todo $y \in S$, o que implica que $x \in \operatorname{MAX}(S, \succcurlyeq)$. Agora, escolha qualquer $x \in \operatorname{MAX}(S, \succcurlyeq)$. Pela definição de $\succcurlyeq$, temos que $x \in c(\{x, y\})$, para todo $y \in S$. Logo, por FAFNIR, $x \in c(S)$. Mostramos, então, que $c(S)=\operatorname{MAX}(S, \succcurlyeq)$. Notamos ainda que, para toda preordem $\hat{\succsim}$, tal que $c(S)=\operatorname{MAX}(S, \hat{\succsim})$ para todo $S \in \Omega_{X}$, devemos ter que $x \hat{\succ} y \Leftrightarrow c(\{x, y\})=\{x\} \Leftrightarrow$ $x \succ y$. Agora, pelo Lema 2, obtemos que existe uma única preordem regular $\succsim$ tal que $c(S)=\operatorname{MAX}(S, \succsim)$, para todo $S \in \Omega_{X}$.

Os Teoremas 3 e 4 indicam que existe um componente residual em AFNIR quando comparado com FAFNIR e TES. De fato, podemos mostrar que AFNIR é equivalente aos axiomas $\alpha$ e $\gamma$ do Sen (1971), e mais uma propriedade ${ }^{1}$ :

Axioma 5. Para todo $S \in \Omega_{X}$ e $x \in S$, se $x \in c(T \cup\{x\})$, para todo $T \subseteq c(S)$ com $T \in \Omega_{X}$, então $x \in c(S)$.

Por outro lado, FAFNIR é exatamente equivalente aos axiomas $\alpha$ e $\gamma$. Portanto, o resíduo supracitado está contido no postulado acima. Não é difícil provar que o resíduo exato é dado pela seguinte propriedade:

Axioma 6. Para todo $S \in \Omega_{X}$, se $c(\{x, z\})=\{x\}$ para algum $x \in S \backslash c(S)$ e $z \in S$, então existe $y \in c(S)$ tal que $c(\{x, y\})=\{y\}$.

Do ponto de vista formal, a correspondência de escolha $c$ satisfaz AFNIR se, e somente se, ela satisfaz FAFNIR e o Axioma 6. Finalmente, quando $\alpha$ e $\gamma$ são verdade, é possível demonstrar que TES é equivalente ao axioma $\delta$ de Sen $(1971)^{2}$.

\footnotetext{
${ }^{1} \mathrm{O}$ axioma $\alpha$ diz que, para qualquer $S, T \in \Omega_{X}$, se $x \in T \subseteq S$ e $x \in c(S)$, então $x \in c(T)$, enquanto que o axioma $\gamma \operatorname{diz}$ que se $\mathcal{M} \subseteq \Omega_{X}, \bigcup\{S: S \in \mathcal{M}\}=: V \in \Omega_{X}$ e $x \in c(S)$ para todo $S \in \mathcal{M}$, então $x \in c(V)$.

${ }^{2} \mathrm{O}$ axioma $\delta$ afirma que para todo par de conjuntos finitios $S, T \in \Omega_{X}$ e para qualquer par $x, y \in c(S)$ com $x \neq y$, se $S \subseteq T$, então $c(T) \neq\{x\}$.
} 


\section{Capítulo 5}

\section{Conclusão}

Nesta dissertação, revisitamos a noção de preordem regular introduzida por Eliaz and Ok (2006). Esperamos ter mostrado que a regularidade é completamente não restritiva para o processo de racionalização em si. Também argumentamos que regularidade pode ser interpretada como exigindo que a preordem seja maximal, dada sua parte estrita. É essa maximalidade que permite com que uma preordem regular, quando utilizada para racionalizar uma correspondência de escolha, identifique nossa noção de indiferença comportamental com a sua parte simétrica e a noção de incomparabilidade observável de Eliaz e Ok com a classe dos seus elementos incomparáveis. Essa é a essência da regularidade: permitir com que a preordem utilizada no processo de racionalização consiga distinguir, de uma forma relevante, entre indiferença e incomparabilidade.

A análise feita por Eliaz and Ok (2006) depende de uma definição incomparabilidade observável. Acreditamos, no entanto, que a noção de indiferença comportamental é mais básica e, por isso, demos prioridade a essa noção. Enquanto que no framework de Eliaz e Ok, essas noções são complementares, a noção de indiferença comportamental tem um maior espectro de aplicações. Para justificar essa última afirmação, mostramos como essa noção pode ser usada para obter representações de models of behavior nas quais quando dois elementos são tratados como comportamentalmente indiferentes, eles não só são ditos indiferentes pelas preferências dos agentes, mas também eles são tratados, de um ponto de vista psicológico, como indistinguíveis. Seria interessante ver qual seria a implicação de nossa noção de indiferença comportamental para a racionalização de outros modelos de decisão, dentre eles o revealed attention model de Masatlioglu et al. (2012) e o endogenous reference model de Ok et al. (2015), por exemplo.

Notamos ainda que para o tipo de representação estudado nesta dissertação, apenas a parte estrita da relação binária $\succsim$ utilizada no processo de racionalização importa, no sentido de que qualquer outra relação binária que compartilhe a mesma parte estrita pode também ser usada para representar a correspondência de escolha. Em particular, a relação $\succeq$, definida por $x \succeq y$ se, e somente se, $y$ não é estritamente preferível a $x$ de acordo com $\succsim$, racionaliza a mesma preferência de escolha que $\succsim$. A relação $\succeq$ é 
completa, mas apenas sua parte estrita é transitiva. Ou seja, $\succeq$ é apenas quase-transitiva. Sen (1971), Schwartz (1976), e Bandyopadhyay and Sengupta (1993) provaram uma série de teoremas de representação que caracterizam correspondências de escolha que podem ser racionalizada por uma relação completa e quase-transitiva. O Lema 2 acima mostra que todos esses resultados poderiam ser reformulados em termos de uma preordem regular.

No entanto, se acreditamos que é importante, não só racionalizar uma correspondência de escolha, mas também capturar outros aspectos observáveis do comportamento dos agentes, a racionalização por uma preordem completa e quase-transitiva, ou mesmo por qualquer outra preordem que não seja regular, não é, de modo algum, equivalente à racionalização por uma preordem que o seja. 


\section{Referências Bibliográficas}

Bandyopadhyay, T. and K. Sengupta (1993). Characterization of generalized weak orders and revealed preference. Economic Theory 3(571-576).

Cherepanov, V., T. Feddersen, and A. Sandroni (2013). Rationalization. Theoretical Economics.

Eliaz, K. and E. A. Ok (2006). Indifference or indecisiveness? choice-theoretic foundations of incomplete preferences. Games and Economic Behavior 56, 61-86.

Masatlioglu, Y., D. Nakajima, and E. Y. Ozbay (2012). Revealed attention. American Economic Review 102(5), 2183-2205.

Ok, E. A., P. Ortoleva, and G. Riella (2015). Revealed (p)reference theory. American Economic Review 105(1), 299-321.

Schwartz, T. (1976). Choice functions, "rationality" conditions, and variations on the weak axiom of revealed preference. Journal of Economic Theory 13(3), 414-427.

Sen, A. K. (1971). Choice functions and revealed preference. Review of Economic Studies 38(3), 307-317. 